\title{
coupleCoC+: an information-theoretic co-clustering-based transfer learning framework for the integrative analysis of single-cell genomic data
}

\author{
Pengcheng Zeng ${ }^{1}$ and Zhixiang Lin ${ }^{* 1}$ \\ ${ }^{1}$ Department of Statistics, The Chinese University of Hong Kong, HK
}

April 16, 2021

\begin{abstract}
Technological advances have enabled us to profile multiple molecular layers at unprecedented single-cell resolution and the available datasets from multiple samples or domains are growing. These datasets, including scRNA-seq data, scATAC-seq data and sc-methylation data, usually have different powers in identifying the unknown cell types through clustering. So, methods that integrate multiple datasets can potentially lead to a better clustering performance. Here we propose couple $\mathrm{CoC}+$ for the integrative analysis of single-cell genomic data. couple $\mathrm{CoC}+$ is a transfer learning method based on the information-theoretic co-clustering framework. In couple $\mathrm{CoC}+$, we utilize the information in one dataset, the source data, to facilitate the analysis of another dataset, the target data. couple CoC+ uses the linked features in the two datasets for effective knowledge transfer, and it also uses the information of the features in the target data that are unlinked with the source data. In addition, couple $\mathrm{CoC}+$ matches similar cell types across the source data and the target data. By applying couple $\mathrm{CoC}+$ to the integrative clustering of mouse cortex scATAC-seq data and scRNA-seq data, mouse and human scRNA-seq data, mouse cortex sc-methylation and scRNA-seq data, and human blood dendritic cells scRNA-seq data from two batches, we demonstrate that couple $\mathrm{CoC}+$ improves the overall clustering performance and matches the cell subpopulations across multimodal single-cell genomic datasets. couple $\mathrm{CoC}+$ has fast convergence and it is computationally efficient. The software is available at https://github.com/cuhklinlab/coupleCoC_plus.
\end{abstract}

Keywords: Transfer learning, Information-theoretic co-clustering, Single-cell genomics

*Corresponding author: zhixianglin@cuhk.edu.hk 


\section{Introduction}

The advances in single-cell technologies have enabled the profiling of multiple molecular layers and have provided great opportunities to study cellular heterogeneity. These technologies include single-cell RNA sequencing (scRNA-seq) that profiles transcription, single-cell ATAC sequencing (scATAC-seq) that profiles accessible chromatin regions (Buenrostro et al., 2015; Mezger et al., 2018: Macaulay et al. 2017), single-cell methylation assays that profile methylated regions (Guo et al. 2013; Smallwood et al., 2014; Clark et al., 2017; Luo et al., 2017) and other methods. The datasets (Rotem et al., 2015; Cusanovich et al., 2015; Rozenblatt-Rosen et al., 2017) brought by these technologies lead to increasing demands for computationally efficient methods for processing and analyzing the data. However, single-cell genomics data often have high technical variation and high noise level due to the minimal amount of genomic materials isolated from individual cells (Kharchenko et al., 2014; Lun et al., 2016; Vallejos et al., 2017; Hicks et al., 2018). These experimental factors bring the challenge of analyzing single-cell genomic data, and affect the results and interpretation of unsupervised learning methods, including dimension reduction and clustering (Jaitin et al., 2014; Usoskin et al., 2015; Lafon and Lee, 2006; Vandermaaten, 2008).

Clustering methods, which group similar cells into sub-populations, are often used as the first step in the analysis of single-cell genomic data. Most clustering methods are designed for clustering one type of measurement. The clustering methods for scRNA-seq data include SIMLR (Wang et al., 2017), SC3 (Kiselev et al., 2017), DIMM-SC (Sun et al., 2017), SAFE-clustering (Yang et al., 2018), SOUP (Zhu et al., 2019), SAME-clustering Huh et al. (2020) and SHARP Wan et al. (2020). The methods chromVAR Schep et al. (2017), scABC Zamanighomi et al. (2018), SCALE (Xiong et al., 2019), cis Topic (Gonzalez-Blas et al., 2019) and Cusanovich2018 (Cusanovich and the others, 2018) are developed for analyzing scATAC-seq data. Clustering methods have also been proposed for methylation data (Kapourani and Sanguinetti, 2018, 2019). To comprehensively analyze the complex biological processes, we need to acquire and integrate different types of measurement from multiple experiments. In recent years, some methods are developed for this purpose. They include Seurat (Butler et al., 2018; Stuart et al., 2019), MOFA (Argelaguet et al., 2018), coupleNMF (Duren et al., 2018), scVDMC (Zhang et al., 2018), LIGER (Welch et al., 2019), scACE (Lin et al., 2019), MOFA+ (Argelaguet et al., 2020), scAI (Jin et al., 2020), couple CoC (Zeng and Lin, 2020) and scMC (Zhang and Nie, 2021). A more comprehensive discussion on integration of single-cell genomic data is presented in David et al. (2020).

To link data from different sources in a way that is biologically meaningful is the most important challenge in the integration of single-cell data across different types of measurement. As an example, we consider the setting where scRNA-seq and scATAC-seq are profiled on similar cell subpopulations but different cells. It is desirable to utilize the information in scRNA-seq data to help us cluster scATAC-seq data, which is typically sparser and noisier. A subset of features in scATAC-seq data are linked with scRNA-seq data, because promoter accessibility/gene activity score are directly linked with gene expression. The linked features help us connect the two data types, which may lead to improvement in clustering scATAC-seq data. Besides the linked features, we can also leverage the unlinked features in the scATAC-seq data: accessibility of the peaks distant from the genes is not directly linked with gene expression in scRNA-seq data. Incorporating more information by including the unlinked features is expected to further improve the clustering performance of the scATAC-seq data. 
In this work, we propose couple $\mathrm{CoC}+$, which is based on the information-theoretic co-clustering (Dhillon et al., 2003) transfer learning framework for the integrative analysis of single-cell genomic data (Fig. 1). The goal of couple $\mathrm{CoC}+$ is to utilize one dataset, the source data (S), to facilitate the analysis of another dataset, the target data. Depending on whether the features are linked with the source data or not, the target data can be partitioned into two parts, data $\mathrm{T}$ with the linked features, and data $U$ with the unlinked features (Fig. 1(a)). As an example, we may use scRNA-seq data as the source data $\mathrm{S}$ and scATAC-seq data as the target data. Data $\mathrm{T}$ is the data matrix of gene activity score, which are directly linked with gene expression in scRNA-seq data, and data $U$ is the data matrix for the accessibility of peaks distal to the genes, which are not directly linked with gene expression. couple $\mathrm{CoC}+$ not only transfers information from the source data, but also utilizes information from the unlinked features in data $\mathrm{U}$. In couple CoC+, both the genomic features and the cells are clustered (Fig. 1(b)). The key for knowledge transfer between the source data and the target data is that the cluster assignments for the linked features are the same. couple $\mathrm{CoC}+$ also performs matching of a subset of cell clusters across the source data and the target data, which may represent shared cell types across the two datasets. We refer our model as couple $\mathrm{CoC}+$, because it is based on the framework of our previously proposed couple CoC (Zeng and $\mathrm{Lin}, 2020$ ). couple $\mathrm{CoC}+$ addresses the limitations of couple $\mathrm{CoC}$ by including the unlinked features in target data and it also integrates co-clustering and cell type matching in one step for better use of information from the source data.

\section{Materials and methods}

In this section, we first introduce the information-theoretic co-clustering framework for source data (Dhillon et al., 2003), and then extend it to our framework of co-clustering source data and target data simultaneously. We will choose the less noisy dataset as the source data, such as scRNA-seq data, and we will choose the noisier dataset as the target data, such as scATACseq data and sc-methylation data. We assume that a subset of features in the target data are linked with the source data: gene activity score in scATAC-seq data and gene body methylation in sc-methylation data are linked with gene expression in scRNA-seq data; and the other subset of features are not directly linked: peak accessibility in scATAC-seq data and DNA methylation levels at non-CG sites for non-overlapping bins in sc-methylation data are not directly linked with the genes in scRNA-seq data. Promoter accessibility may also be used to link scATAC-seq data with scRNA-seq when gene activity score is not available. Promoter accessibility may have less power in separating the cell types compared with gene activity score, because gene activity score incorporates more regions nearby the gene. We expect to improve the clustering performance of the target data by transferring knowledge from the source data via the linked features and also effectively utilizing the information in the unlinked features in the target data.

\section{Information-theoretic co-clustering}

We first consider the source data. Let $\mathrm{S}$ be a $n_{\mathrm{S}}$ by $q$ matrix representing this dataset with $q$ features for $n_{\mathrm{S}}$ cells. Let $X$ and $Z_{\mathrm{S}}$ be discrete random variables, representing the possible outcome of cell labels and feature labels, respectively. $X$ takes values from the set $\left\{1,2, \ldots, n_{\mathrm{S}}\right\}$ and $Z_{\mathrm{S}}$ takes values from the set $\{1,2, \ldots, q\}$. We let $p_{\mathrm{S}}\left(X, Z_{\mathrm{S}}\right)$ be the joint probability distribution 


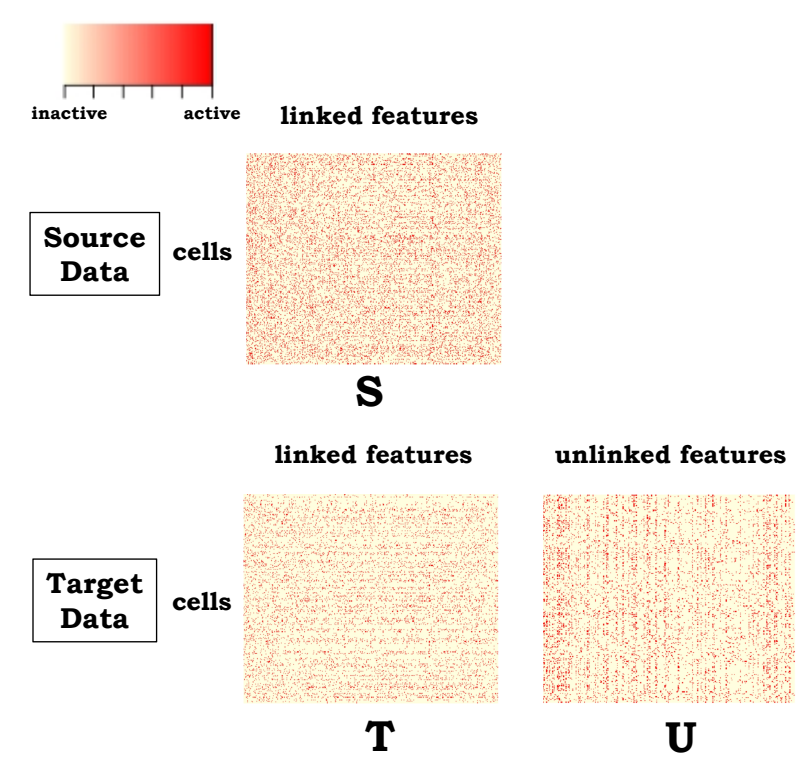

(a). The input data matrices

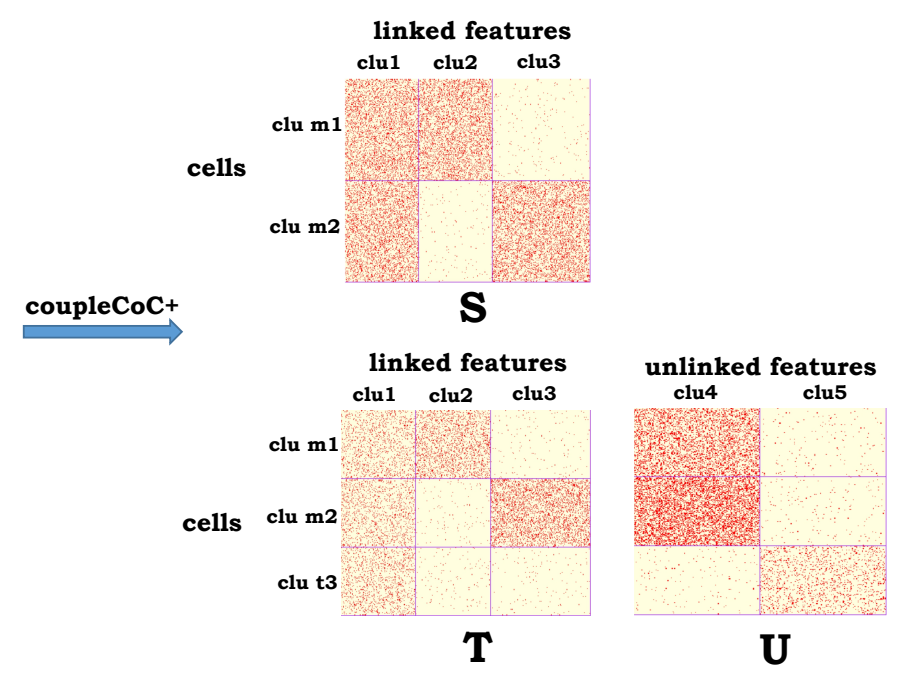

(b). The clustering results

Figure 1: Toy example of our proposed coupleCoC+. (a). Source data is represented by "S". Based on whether the features are linked with those in the source data, we partition the target data into two parts, "T" and "U". The features in data T are linked with data S, while the features in data $U$ are not directly linked with data $S$. The cells in data $\mathrm{T}$ and $\mathrm{U}$ are the same. Red color means that the corresponding features are active, and yellow color means that they are inactive. (b). The clustering results by couple $\mathrm{CoC}+$. couple $\mathrm{CoC}+$ co-clusters the data $\mathrm{S}, \mathrm{T}$ and U simultaneously by clustering similar cells and similar features. A subset of the cell clusters are also matched between the source data and the target data, representing shared cell types. "clu" is the abbreviation of "cluster", and "m" means the matched clusters. "clu t" represents the cell cluster that is unique to the the target data.

for $X$ and $Z_{\mathrm{S}}$, and define $p_{\mathrm{S}}\left(X=x, Z_{\mathrm{S}}=z\right)$ as the probability of the $z$-th feature being active in the $x$-th cell: the more active the feature, the higher the value. This joint probability is estimated from the normalized dataset, i.e. scaling the data matrix $\mathrm{S}$ to have total sums equal to 1 , and we have $p_{\mathrm{S}}\left(X=x, Z_{\mathrm{S}}=z\right)=\frac{\mathrm{S}_{x z}}{\sum_{x=1}^{n_{\mathrm{S}}} \sum_{z=1}^{q} \mathrm{~S}_{x z}}$, where $x \in\left\{1, \ldots, n_{\mathrm{S}}\right\}, z \in\{1, \ldots, q\}$.

The goal of co-clustering is to cluster similar cells into clusters and similar features into clusters. Assume that we want to cluster the cells into $N_{\mathrm{S}}$ clusters, and the features into $K$ clusters. We denote the clusters of cells and features as the possible outcomes of discrete random variables $\tilde{X}$ and $\tilde{Z}_{\mathrm{S}}$, where $\tilde{X}$ and $\tilde{Z}_{\mathrm{S}}$ take values from the sets of cell cluster indexes $\left\{1, \ldots, N_{\mathrm{S}}\right\}$ and feature cluster indexes $\{1, \ldots, K\}$, respectively. To map cells to cell clusters and features to feature clusters, we use $C_{X}(\cdot)$ and $C_{Z}(\cdot)$ to represent the clustering functions of cells and features, respectively. $C_{X}(x)=\tilde{x}\left(\tilde{x}=1, \ldots, N_{\mathrm{S}}\right)$ indicates that cell $x$ belongs to cluster $\tilde{x}$, and $C_{Z}(z)=\tilde{z}$ $(\tilde{z}=1, \ldots, K)$ indicates that feature $z$ belongs to cluster $\tilde{z}$. We then let $\tilde{p}_{\mathrm{S}}\left(\tilde{X}, \tilde{Z}_{\mathrm{S}}\right)$ be the joint 
probability distribution for $\tilde{X}$ and $\tilde{Z}_{\mathrm{S}}$, and this distribution can be expressed as

$$
\tilde{p}_{\mathrm{S}}\left(\tilde{X}=\tilde{x}, \tilde{Z}_{\mathrm{S}}=\tilde{z}\right)=\sum_{x \in\left\{C_{X}(x)=\tilde{x}\right\}} \sum_{z \in\left\{C_{Z}(z)=\tilde{z}\right\}} p_{\mathrm{S}}\left(X=x, Z_{\mathrm{S}}=z\right) .
$$

Note that $\tilde{p}_{\mathrm{S}}\left(\tilde{X}=\tilde{x}, \tilde{Z}_{\mathrm{S}}=\tilde{z}\right)$ is connected to $p_{\mathrm{S}}\left(X, Z_{\mathrm{S}}\right)$ via the clustering functions $C_{X}(\cdot)$ and $C_{Z}(\cdot)$. The matrix $\tilde{p}_{\mathrm{S}}\left(\tilde{X}, \tilde{Z}_{\mathrm{S}}\right)_{N_{\mathrm{S}} \times K}$ can be interpreted as the low dimension representations for the cell clusters in the source data $\mathrm{S}$.

The information-theoretic co-clustering (Dhillon et al., 2003) aims at finding the optimal clustering functions $C_{X}(\cdot)$ and $C_{Z}(\cdot)$ to minimize the loss of mutual information:

$$
\ell_{\mathrm{S}}\left(C_{X}, C_{Z}\right)=I\left(X ; Z_{\mathrm{S}}\right)-I\left(\tilde{X} ; \tilde{Z}_{\mathrm{S}}\right)
$$

where $I(\cdot)$ denotes the function of mutual information, and we have

$I\left(X ; Z_{\mathrm{S}}\right)=\sum_{x} \sum_{z} p_{\mathrm{S}}(x, z) \log \frac{p_{\mathrm{S}}(x, z)}{p_{\mathrm{S}}(x) p_{\mathrm{S}}(z)}$, and $I\left(\tilde{X} ; \tilde{Z}_{\mathrm{S}}\right)=\sum_{\tilde{x}} \sum_{\tilde{z}} \tilde{p}_{\mathrm{S}}(\tilde{x}, \tilde{z}) \log \frac{\tilde{p}_{\mathrm{S}}(\tilde{x}, \tilde{z})}{\tilde{p}_{\mathrm{S}}(\tilde{x}) \tilde{p}_{\mathrm{S}}(\tilde{z})}$.

\section{The framework of couple CoC+}

We now extend the information-theoretic co-clustering framework to multiple datasets, and simultaneously perform matching of the cell types across datasets (see the toy example in Fig. 1).

Besides the source data $\mathrm{S}$, we have another target data. The goal of couple CoC+ is to improve the clustering performance of the target data, utilizing the information in the source data. Depending on whether the features are linked with the source data, the target data can be partitioned into two parts: data $\mathrm{T}$, which includes the linked features; and data U, which includes the unlinked features. Similar to the corresponding definitions for source data, we have the loss of mutual information for co-clustering the data $\mathrm{T}$ :

$$
\ell_{\mathrm{T}}\left(C_{Y}, C_{Z}\right)=I\left(Y_{\mathrm{T}} ; Z_{\mathrm{T}}\right)-I\left(\tilde{Y}_{\mathrm{T}} ; \tilde{Z}_{\mathrm{T}}\right),
$$

where $Y_{\mathrm{T}}$ and $Z_{\mathrm{T}}$ are the discrete random variables representing the cell labels and the feature labels in data $\mathrm{T}$, respectively. We have $I\left(Y_{\mathrm{T}} ; Z_{\mathrm{T}}\right)=\sum_{y} \sum_{z} p_{\mathrm{T}}(y, z) \log \frac{p_{\mathrm{T}}(y, z)}{p_{\mathrm{T}}(y) p_{\mathrm{T}}(z)}$. $\tilde{Y}_{\mathrm{T}}$ and $\tilde{Z}_{\mathrm{T}}$ are the discrete random variables representing the cell cluster labels and the feature cluster labels in data $\mathrm{T}$, respectively. We have $I\left(\tilde{Y}_{\mathrm{T}} ; \tilde{Z}_{\mathrm{T}}\right)=\sum_{\tilde{y}} \sum_{\tilde{z}} \tilde{p}_{\mathrm{T}}(\tilde{y}, \tilde{z}) \log \frac{\tilde{p}_{\mathrm{T}}(\tilde{y}, \tilde{z})}{\tilde{p}_{\mathrm{T}}(\tilde{y}) \tilde{p}_{\mathrm{T}}(\tilde{z})} \cdot C_{Y}$ and $C_{Z}$ are the clustering functions for the cells and the features in data $\mathrm{T}$, respectively. Note that we assume that the feature clustering function $C_{Z}$ is the same for the linked features in data $\mathrm{S}$ and T. The function $C_{Z}$ is the key for knowledge transfer between source data and target data. By clustering similar features using the information from the source data, it effectively reduces the noise in the target data.

We also have the loss of mutual information for co-clustering the data U:

$$
\ell_{\mathrm{U}}\left(C_{Y}, C_{U}\right)=I\left(Y_{\mathrm{U}} ; Z_{\mathrm{U}}\right)-I\left(\tilde{Y}_{\mathrm{U}} ; \tilde{Z}_{\mathrm{U}}\right),
$$

where $Y_{\mathrm{U}}$ and $Z_{\mathrm{U}}$ are the discrete random variables representing the cell labels and the feature labels in data $\mathrm{U}$, respectively. We have $I\left(Y_{\mathrm{U}} ; Z_{\mathrm{U}}\right)=\sum_{y} \sum_{u} p_{\mathrm{U}}(y, u) \log \frac{p_{\mathrm{U}}(y, u)}{p_{\mathrm{U}}(y) p_{\mathrm{U}}(u)}$. $\tilde{Y}_{\mathrm{U}}$ and $\tilde{Z}_{\mathrm{U}}$ are the discrete random variables representing the cell cluster labels and the feature cluster labels in data $\mathrm{U}$, respectively. We have $I\left(\tilde{Y}_{\mathrm{U}} ; \tilde{Z}_{\mathrm{U}}\right)=\sum_{\tilde{y}} \sum_{\tilde{u}} \tilde{p}_{\mathrm{U}}(\tilde{y}, \tilde{u}) \log _{\tilde{p}_{\mathrm{U}}(\tilde{y}) \tilde{p}_{\mathrm{U}}(\tilde{u})} \cdot C_{Y}$ and $C_{U}$ are the clustering functions for the cells and the features in data $U$, respectively. Because the cells in data $\mathrm{U}$ and $\mathrm{T}$ are the same, data $\mathrm{U}$ and $\mathrm{T}$ share the same cell clustering function $C_{Y}$.

The matrices $\tilde{p}_{\mathrm{S}}\left(\tilde{X}, \tilde{Z}_{\mathrm{S}}\right)$ and $\tilde{p}_{\mathrm{T}}\left(\tilde{Y}_{\mathrm{T}}, \tilde{Z}_{\mathrm{T}}\right)$ can be interpreted as the low dimension representations 
for the cell clusters in the source data and the target data. A subset of the clusters in the source data and target data may be matched, representing similar cell types across the two datasets. We denote $h_{\mathrm{T}, N_{\text {sub }}}$ as a permutation of size $N_{\text {sub }}$ for the indexes of the cell clusters in data T, and denote $h_{\mathrm{S}, N_{\mathrm{sub}}}$ as an ordered permutation of size $N_{\tilde{\tilde{Z}}}$ for the indexes of the cell clusters in source data $\mathrm{S}$. We then use $D_{\mathrm{KL}}\left(\hat{p}_{\mathrm{T}}\left(\tilde{Y}_{h_{\mathrm{T}, N_{\mathrm{sub}}}}, \tilde{Z}_{\mathrm{T}}\right) \| \hat{p}_{\mathrm{S}}\left(\tilde{X}_{h_{\mathrm{S}, N_{\mathrm{sub}}}}, \tilde{Z}_{\mathrm{S}}\right)\right)$ to measure the statistical distance between two probability distributions $\hat{p}_{\mathrm{T}}\left(\tilde{Y}_{h_{\mathrm{T}, N_{\mathrm{cuh}}}}, \tilde{Z}_{\mathrm{T}}\right)$ and $\hat{p}_{\mathrm{S}}\left(\tilde{X}_{h_{\mathrm{S}, N_{\mathrm{sub}}}}, \tilde{Z}_{\mathrm{S}}\right)$, where $D_{\mathrm{KL}}(\cdot \| \cdot)$ is KullbackLeibler (KL) divergence (Cover and Thomas, 1991). These two distributions are obtained by extracting the rows $h_{\mathrm{T}, N_{\text {sub }}}$ and $h_{\mathrm{S}, N_{\mathrm{sub}}}$ from $\tilde{p}_{\mathrm{T}}\left(Y_{\mathrm{T}}, \tilde{Z}_{\mathrm{T}}\right)$ and $\tilde{p}_{\mathrm{S}}\left(\tilde{X}, \tilde{Z}_{\mathrm{S}}\right)$ correspondingly and then scaling the two submatrices to have summation equal to 1 . The smaller the KL divergence, the more similar the subsets of cell clusters chosen by $h_{\mathrm{T}, N_{\mathrm{sub}}}$ and $h_{\mathrm{S}, N_{\mathrm{sub}}}$.

To co-cluster the three data T, S and U simultaneously, and to match a subset of cell clusters across the source data and the target data, we propose to solve the following optimization problem in couple $\mathrm{CoC}+$ :

$$
\underset{\substack{C_{Y}, C_{X}, C_{Z}, C_{U} \\ h_{\mathrm{T}, N_{\mathrm{sub}}}, h_{\mathrm{S}, N_{\mathrm{sub}}}}}{\operatorname{argmin}}\left(C_{Y}, C_{Z}\right)+\lambda \ell_{\mathrm{S}}\left(C_{X}, C_{Z}\right)+\beta \ell_{\mathrm{U}}\left(C_{Y}, C_{U}\right)+\gamma D_{\mathrm{KL}}\left(\hat{p}_{\mathrm{T}}\left(\tilde{Y}_{h_{\mathrm{T}, N_{\mathrm{sub}}}}, \tilde{Z}_{\mathrm{T}}\right) \| \hat{p}_{\mathrm{S}}\left(\tilde{X}_{h_{\mathrm{S}, N_{\mathrm{sub}}}}, \tilde{Z}_{\mathrm{S}}\right)\right) .
$$

As mentioned before, the two terms $\ell_{\mathrm{T}}\left(C_{Y}, C_{Z}\right)$ and $\ell_{\mathrm{S}}\left(C_{X}, C_{Z}\right)$ in formula (5) share the same feature cluster $C_{Z}$, which can be viewed as a bridge to transfer knowledge between the source data and the target data (Dai et al., 2008; Zeng and Lin, 2020). The dimension of the feature space shared by the source data $\mathrm{S}$ and the data $\mathrm{T}$ is reduced by clustering and aggregating similar features. Aggregating similar features guided by the source data $\mathrm{S}$ enables knowledge transfer between the source data $\mathrm{S}$ and the data $\mathrm{T}$, which reduces the noise in the single-cell data and can generally improve the clustering performance of cells in the target data. The term $\ell_{\mathrm{U}}\left(C_{Y}, C_{U}\right)$ corresponds to the features in the target data that are unlinked with the source data. Incorporating more information from the target data by including more features will also benefit the clustering performance of the target data. The term $D_{\mathrm{KL}}\left(\hat{p}_{\mathrm{T}}\left(\tilde{Y}_{h_{\mathrm{T}, N_{\mathrm{sub}}}}, \tilde{Z}_{\mathrm{T}}\right) \| \hat{p}_{\mathrm{S}}\left(\tilde{X}_{h_{\mathrm{S}, N_{\mathrm{sub}}}}, \tilde{Z}_{\mathrm{S}}\right)\right)$ further borrows information from the source data for the matched cell clusters. $\lambda$ is a hyperparameter that controls the contribution of the source data $\mathrm{S}, \beta$ is a hyperparameter that controls the contribution of the unlinked features in the target data, and $\gamma$ is a hyperparameter that controls the contribution of cell types matching across the source data $\mathrm{S}$ and the data $\mathrm{T}$.

The optimization problem (5) can be solved by iteratively updating $C_{X}, C_{Y}, C_{Z}, C_{U}, h_{\mathrm{T}, N_{\mathrm{sub}}}$ and $h_{\mathrm{S}, N_{\mathrm{sub}}}$. The technical details of the updates are presented in Sections 1.1 and 1.2 in the Supplementary Materials. The objective function in the optimization problem (5) is non-increasing in the updates of $C_{Y}, C_{X}, C_{Z}, C_{U}, h_{\mathrm{T}, N_{\mathrm{sub}}}$ and $h_{\mathrm{S}, N_{\mathrm{sub}}}$, and the algorithm will converge to a local minimum. Finding the global optimal solution is NP-hard. The algorithm converges in a finite number of iterations due to the finite search space (see details in Section: Convergence and running time). In practice, this algorithm works well in real single-cell genomic data analysis.

Lastly, we note that the major differences between couple $\mathrm{CoC}$ (Zeng and Lin, 2020) and couple $\mathrm{CoC}+$ lie in two aspects: (a). couple $\mathrm{CoC}$ does not include the unlinked features in the target data. We will demonstrate through the real data examples that incorporating more information by including the unlinked features will benefit clustering of the target data. (b). In couple CoC, cell type matching is a separate step from co-clustering. In couple $\mathrm{CoC}+$, we simultaneously perform cell type matching and co-clustering. Merging cell type matching and co-clustering in one step 
can leverage more information from the source data.

\section{Choosing source data and target data}

In couple $\mathrm{CoC}+$, the dataset that is less sparse and less noisy should be chosen as the source data, and dataset that is more sparse and noisier should be chosen as the target data. By doing so, we expect to borrow more useful information from the source data to clustering the target data. Based on this rule, we generally choose scRNA-seq data as the source data and choose scATACseq data or sc-methylation data as the target data. In practice, we utilize the proportion of zero entries in the data matrix to evaluate the sparsity, and it is calculated as:

$$
\frac{\# \text { of zero entries }}{\text { \#of cells } \times \text { \# of features }} \text {. }
$$

We will describe the details on how to choose the source data and the target data case-by-case in the real data examples.

\section{Feature selection}

Features that are directly related to the genes are used as linked features across datasets: we use gene activity score (prefered) or promoter accessibility in scATAC-seq data, homologs in human and mouse scRNA-seq data, and gene body methylation in sc-methylation data. Features that are not directly linked to genes are treated as the unlinked features in target data: we use accessibility of peak values in scATAC-seq data and DNA methylation levels at non-CG sites for non-overlapping 100kb bins in sc-methylation data. We use the mouse specific genes that are not included in human as the unlinked features when we use mouse scRNA-seq data as the target data and human scRNA-seq data as the source data. We implement feature selection before performing clustering. We use the $\mathrm{R}$ toolkit Seurat (Butler et al., 2018; Stuart et al., 2019) to select 1000 most variable features for each data $\mathrm{S}, \mathrm{T}$ and $\mathrm{U}$.

\section{Data preprocessing}

We take log transformation for scRNA-seq data to alleviate the effect of extreme values in the data matrices: we use $\log 2(\mathrm{TPM}+1)$ for TPM data and $\log 2(\mathrm{UMI}+1)$ for UMI data as the input. We use gene activity score or promoter accessibility and binarized count for peaks as the input for scATAC-seq data. We use DNA methylation levels at non-CG sites in the gene body and non-overlapping $100 \mathrm{~kb}$ bins as the input for sc-methylation data. We impute the missing values in sc-methylation data with the overall mean. Since the relationship between gene body methylation and gene expression is negative, we further transform sc-methylation data by 1-methylation level. Our proposed couple CoC + can automatically adjust for sequencing depth, so we do not need to normalize for sequencing depth. The input formats of data S, T and U are described case-by-case in real data examples. 


\section{Hyperparameter selection}

Before implementing the couple CoC+ algorithm, we use the Calinski-Harabasz (CH) index (Calinski and Harabasz, 1974) to pre-determine the number of cell clusters $N_{\mathrm{T}}$ for target data and the number of cell clusters $N_{\mathrm{S}}$ for source data separately. $\mathrm{CH}$ index is proportional to the ratio of between-clusters dispersion and within-cluster dispersion:

$$
f(N)=\frac{S S_{B}(N)}{S S_{W}(N)} \times \frac{n-N}{N-1},
$$

where $S S_{B}(N)$ is the overall between-cluster variance, and $S S_{W}(N)$ is the overall within-cluster variance, $N$ is the number of cell clusters, and $n$ is the total number of cells. For each cluster number $N$, we first cluster the dataset by minimizing $\ell_{\mathrm{T}}$ for target data (or $\ell_{\mathrm{S}}$ for source data) by $\mathrm{CoC}$ (i.e. information theoretic co-clustering algorithm in Dhillon et al. (2003), which is equivalent to setting $\lambda=\beta=\gamma=0$ in formula (5)), and then calculate $S S_{B}(N), S S_{W}(N)$, and obtain $f(N)$. We choose the number of cell clusters $N$ with the highest $\mathrm{CH}$ index.

Our coupleCoC + is an unsupervised learning model, and it is hard to determine the value of non-negative hyperparameters $\lambda, \beta$ and $\gamma$, and the number of feature clusters $K$ in data T and $K_{0}$ in data $\mathrm{U}$ in theory. In practice, we tune these parameters empirically on the datasets themselves by optimizing the $\mathrm{CH}$ index. Let $\Omega \triangleq\left(\lambda, \beta, \gamma, K, K_{0}\right)$, and we use grid search to choose the best combination of hyperparameters that has the highest $\mathrm{CH}$ index for the target data: $f_{\mathrm{T}}(\Omega)=\frac{S S_{B}(\Omega)}{S S_{W}(\Omega)} \times \frac{n_{\mathrm{T}}-N_{\mathrm{T}}}{N_{\mathrm{T}}-1}$. We choose the search domains $\lambda, \beta, \gamma \in(0,5)$ and $K, K_{0} \in(1,20)$. Grid search performs well in real data analysis.

The value of $N_{\text {sub }}$ can be user-defined or chosen heuristically. The intuition for choosing $N_{\text {sub }}$ is that the KL divergence $D_{\mathrm{KL}}\left(\hat{p}_{\mathrm{T}}\left(\tilde{Y}_{h_{\mathrm{T}, N_{\mathrm{sub}}}}, \tilde{Z}_{\mathrm{T}}\right) \| \hat{p}_{\mathrm{S}}\left(\tilde{X}_{h_{\mathrm{S}, N_{\mathrm{sub}}}}, \tilde{Z}_{\mathrm{S}}\right)\right)$ will be larger if the clusters being matched are less similar when they are forced to be matched. $N_{\text {sub }}$ is chosen similarly as in Zeng and Lin (2020). More details are given in Section 1.3 in Supplementary Materials. Though there

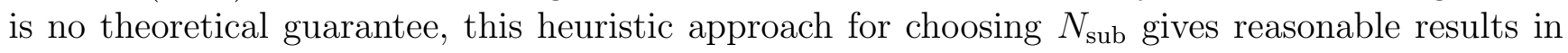
the real data examples.

\section{Evaluation metrics}

We evaluate the clustering performance by normalized mutual information (NMI) and adjusted Rand index (ARI) (Christopher et al., 2008). Assume that $G$ is the known ground-truth labels of cells and $Q$ is the predicted clustering assignments, then NMI is calculated as:

$$
\frac{I(Q ; G)}{\sqrt{H(Q) * H(G)}},
$$

where $\mathrm{H}$ is the entropy. $\mathrm{NMI}$ is normalized mutual information score and takes value between 0 and 1. Assume that $n$ is the total number of single cells, $n_{Q, i}$ is the number of cells assigned to the $i$-th cluster in $Q, n_{G, j}$ is the number of cells belonging to the $j$-th cell type in $G$, and $n_{i, j}$ is the number of overlapping cells between the $i$-th cluster in $Q$ and the $j$-th cell type in $G$. As a corrected-for-chance version of the Rand index, ARI is calculated as:

$$
\frac{\sum_{i j}\left(\begin{array}{c}
n_{i, j} \\
2
\end{array}\right)-\left[\sum_{i}\left(\begin{array}{c}
n_{Q, i} \\
2
\end{array}\right) \sum_{j}\left(\begin{array}{c}
n_{G, j} \\
2
\end{array}\right) /\left(\begin{array}{c}
n \\
2
\end{array}\right)\right]}{\frac{1}{2}\left[\sum_{i}\left(\begin{array}{c}
n_{Q, i} \\
2
\end{array}\right)+\sum_{j}\left(\begin{array}{c}
n_{G, j} \\
2
\end{array}\right)\right]-\left[\sum_{i}\left(\begin{array}{c}
n_{Q, i} \\
2
\end{array}\right) \sum_{j}\left(\begin{array}{c}
n_{G, j} \\
2
\end{array}\right)\right] /\left(\begin{array}{c}
n \\
2
\end{array}\right)} .
$$


The higher values of NMI and ARI indicate better clustering performance.

\section{Results}

We evaluated our method couple $\mathrm{CoC}+$ in four real data examples, including one example for clustering mouse cortex scATAC-seq data and scRNA-seq data, one example for clustering human and mouse scRNA-seq data, one example for clustering mouse cortex sc-methylation data and scRNA-seq data, and one example for clustering human blood dendritic cells scRNA-seq data generated from two experimental batches. UMAP visualizations of all these raw data are presented in Fig.S.1-S.4. We compared coupleCoC+ with coupleCoC (Zeng and Lin, 2020), CoC (Dhillon et al. 2003), $k$-means and other commonly used clustering methods for single-cell genomic data, including SC3 (Kiselev et al., 2017), SIMLR (Wang et al., 2017), SAME-clustering (Huh et al., 2020) and SHARP (Wan et al., 2020) for scRNA-seq data, Cusanovich2018 (Cusanovich and the others, 2018) and cis Topic (Gonzalez-Blas et al., 2019) for scATAC-seq data (we implemented louvain clustering after dimension reduction by Cusanovich2018 (Cusanovich and the others, 2018) and cis Topic (Gonzalez-Blas et al., 2019), which was suggested in a recent benchmark study on scATAC-seq data (Chen et al., 2019) ), BPRMeth-G (Kapourani and Sanguinetti, 2018) (Gaussianbased model proposed in Kapourani and Sanguinetti (2018)) for sc-methylation data, and Seurat (Stuart et al., 2019), LIGER (Welch et al., 2019) and scACE (Lin et al., 2019)for the integrative clustering of source data and target data. For a fair comparison, we implemented the benchmarked methods (except coupleCoC) with both the linked and the unlinked features. We determined the number of cell clusters for couple $\mathrm{CoC}+$ by the $\mathrm{CH}$ index, and we used the true number of cell clusters for the other methods, except for the methods Seurat (Stuart et al., 2019) and LIGER (Welch et al. 2019), which automatically determine the number of cell clusters. We used ARI, NMI and the clustering table to evaluate the clustering results, where the cell type labels provided in their original publications were treated as the ground truth.

\section{Example 1: integrative clustering for mouse cortex scATAC-seq data and scRNA-seq data}

We first evaluated couple CoC + by jointly clustering mouse cortex scATAC-seq data (Cusanovich and the others, 2018) and scRNA-seq data (Tasic et al., 2018). We collected 458 oligodendrocytes, 551 astrocytes, 319 inhibitory neurons, 197 microglia cells for the scATAC-seq data and collected six subtypes of inhibitory neurons (including 1122 Lamp5 cells, 1741 Sst cells, 1337 Pvalb cells, 125 Sncg cells, 27 Serpinfi cells and 1728 Vip cells), 368 astrocytes and 91 oligodendrocytes for the scRNA-seq data. Note that microglia cells are not used in the scRNA-seq data. We chose the scATAC-seq data as the target data, and scRNA-seq data as the source data, because scATAC-seq data is noisier and sparser (The proportions of zero entries in the scATAC-seq data and scRNA-seq data are $95.71 \%$ and $86.68 \%$, respectively.) We used gene activity score in scATAC-seq data as the features that are linked with scRNA-seq data, and used the accessibility of the peaks as the unlinked features. The input formats are $\log (\mathrm{TPM}+1)$ for scRNA-seq data, and binarized gene activity score and binarized peak accessibility for scATAC-seq, respectively. We used the provided cell type labels as a benchmark for evaluating the performance of the clustering methods. The numbers of cell clusters with the highest $\mathrm{CH}$ indexes are $N_{\mathrm{T}}=5$ for the target data and $N_{\mathrm{S}}=6$ for 
the source data (Fig.S.5). In the source data, there are six subtypes of inhibitory neurons and two other cell types, and the smaller cell cluster number $\left(N_{\mathrm{S}}=6\right)$ chosen by $\mathrm{CH}$ index likely represents the similarity of the six subtypes of inhibitory neurons. We implemented couple CoC + with $N_{\mathrm{S}}=8$ and $N_{\mathrm{T}}=5$. We set the tuning parameters in couple $\mathrm{CoC}+$ as $\lambda=2.5, \beta=0.01, \gamma=1, K=$ $12, K_{0}=6$ by grid search. We set the number of $N_{\text {sub }}$ as 4 , because the objective function $g\left(N_{\text {sub }}\right)$ for choosing $N_{\text {sub }}$ (The formula of $g\left(N_{\text {sub }}\right)$ is given in Section 1.3 in Supplementary Materials) obtains the minimum 0.021 when $N_{\text {sub }}=4$ (Fig.S.6).

Table 1 shows that couple $\mathrm{CoC}+$ performs better than couple $\mathrm{CoC}$ on clustering the target data, because couple $\mathrm{CoC}+$ utilizes information from clustering the data $\mathrm{U}$ which is not present in couple $\mathrm{CoC}$, and it performs much better than $\mathrm{CoC}$, because couple $\mathrm{CoC}+$ transfers knowledge from clustering the source data S. The methods cis Topic and Cusanovich2018 perform well but not as good as couple $\mathrm{CoC}+$. The performance of clustering the source data by couple $\mathrm{CoC}+$ is better than couple $\mathrm{CoC}$, and ranks the third among ten clustering methods. The integrative clustering methods Seurat, LIGER and $s c \mathrm{ACE}$ perform worse than couple $\mathrm{CoC}+$, except for clustering the source data by LIGER. The clustering table (Table.S.1) by coupleCoC+ shows that the cell types astrocytes and oligodendrocytes are matched well across the two data types. Fig. 2 shows the heatmap after clustering by couple $\mathrm{CoC}+$. couple $\mathrm{CoC}+$ clearly clusters similar cells and features. In addition, we can see that the pairs of matched cell clusters m1-4 in the two datasets clearly resemble each other more, compared with the other unmatched cell clusters.

Next we investigated the features that are clustered together by couple CoC+. Feature cluster "clu4" is specific to cell cluster "clu m3" in scRNA-seq and scATAC-seq data, which are mostly oligodendrocyte cells; and feature cluster "clu6" is specific to "clu t5" in scATAC-seq data, which are mostly microglia cells. We performed functional annotation enrichment analysis using DAVID (Huang et al. 2009a b). The genes in feature cluster "clu4" (59 genes in total) are highly enriched for the terms related to myelin (more comprehensive list in Table S.2.). The top three terms and their Bonferroni corrected p-values are ("myelin sheath", $1.44 \times 10^{-11}$ ), ("myelination", $1.12 \times 10^{-7}$ ) and ("structural constituent of myelin sheath", $2.51 \times 10^{-5}$ ), respectively. By creating myelin sheath, oligodendrocytes provide support and insulation to axons in the central nervous system. The genes in feature cluster "clu6" (198 genes in total) are highly enriched for the terms related to the immune system (more comprehensive list in Table S.3.). The top two terms and their Bonferroni corrected $p$-values are ("immunity", $8.27 \times 10^{-22}$ ) and ("immune system process", $3.00 \times 10^{-19}$ ), respectively. Microglia represents a specialized population of macrophages-like cells in the central nervous system (CNS) considered immune sentinels that are capable of orchestrating a potent inflammatory response (Sara et al., 2018). In summary, the genes that are clustered together by couple $\mathrm{CoC}+$ tend to be enriched for functional annotation terms closely related to the cell clusters in which they are active.

\section{Example 2: integrative clustering for mouse and human scRNA-seq data}

In the second example, we examined our couple $\mathrm{CoC}+$ in datasets across different species, i.e. human and mouse scRNA-seq data (Fran et al., 2019). We collected 99 clara cells, 14 ependymal cells, 179 mouse pulmonary alveolar type II in the mouse scRNA-seq dataset, and we collected 113 clara cells and 58 ependymal cells in the human scRNA-seq dataset. Note that there is one cell type in the mouse scRNA-seq data that is not present in the human scRNA-seq data. We 
Table 1: The results of clustering the cells in source data and target data in four examples. Note that the capital letters in the brackets represent the input data matrices for the corresponding methods: S represents source data, T and U represent the sub-matrices for the linked and unlinked features in target data, respectively. For integrative analysis methods (coupleCoC+, coupleCoC, Seurat, LIGER, scACE) that utilize both the source data and the target data as input, they produce clustering results of the cells in source data and target data simultaneously. We then summarize the clustering results by calculating ARI and NMI for source data and target data separately. For the remaining methods that are implemented on only one dataset, they produce clustering results of the cells in source data or target data independently. We then summarize the clustering results by calculating ARI and NMI for source data and target data separately. The source data type is scRNA-seq data for all four examples, while the target data types for examples 1-4 are scATAC-seq data, scRNA-seq data, sc-methylation data and scRNA-seq data, respectively. The symbol "-" means that the corresponding clustering method is not designed for that data type. We only compared the methods for integrative analysis of multiple datasets in example $4 . n_{\mathrm{T}}$ and $n_{\mathrm{S}}$ are the numbers of cells in the target data and the source data, correspondingly. Because we included the unlinked features when implementing CoC, $k$-means, Cusanovich2018, cis Topic, SC3, SIMLR and BPRMeth-G, the clustering results for these methods are better than that presented in Zeng and Lin (2020).

\begin{tabular}{|c|c|c|c|c|c|c|c|c|c|}
\hline & \multirow[t]{2}{*}{ Clustering methods } & \multicolumn{2}{|c|}{$\begin{array}{c}\text { Example } 1 \\
\left(n_{\mathrm{T}}=1525, n_{\mathrm{S}}=6539\right)\end{array}$} & \multicolumn{2}{|c|}{$\begin{array}{c}\text { Example } 2 \\
\left(n_{\mathrm{T}}=292, n_{\mathrm{S}}=171\right)\end{array}$} & \multicolumn{2}{|c|}{$\begin{array}{c}\text { Example 3 } \\
\left(n_{\mathrm{T}}=1102, n_{\mathrm{S}}=2383\right)\end{array}$} & \multicolumn{2}{|c|}{$\begin{array}{c}\text { Example } 4 \\
\left(n_{\mathrm{T}}=288, n_{\mathrm{S}}=288\right)\end{array}$} \\
\hline & & ARI & NMI & ARI & NMI & ARI & NMI & ARI & NMI \\
\hline \multirow{12}{*}{$\begin{array}{c}\text { Target } \\
\text { data }\end{array}$} & couple $\mathrm{CoC}+(\mathrm{T}+\mathrm{S}+\mathrm{U})$ & 0.898 & 0.886 & 0.859 & 0.769 & 0.869 & 0.782 & 0.837 & 0.804 \\
\hline & couple $\mathrm{CoC}(\mathrm{T}+\mathrm{S})$ & 0.843 & 0.850 & 0.790 & 0.672 & 0.619 & 0.500 & 0.838 & 0.800 \\
\hline & $\mathrm{CoC}(\mathrm{T}+\mathrm{U})$ & 0.810 & 0.879 & 0.819 & 0.728 & 0.839 & 0.743 & - & - \\
\hline & $k$-means $(\mathrm{T}+\mathrm{U})$ & 0.479 & 0.615 & 0.636 & 0.537 & 0.738 & 0.638 & - & - \\
\hline & Cusanovich2018 $(\mathrm{T}+\mathrm{U})$ & 0.876 & 0.824 & - & - & - & - & - & - \\
\hline & cis Topic $(\mathrm{T}+\mathrm{U})$ & 0.860 & 0.855 & - & - & - & - & - & - \\
\hline & $\mathrm{SC} 3(\mathrm{~T}+\mathrm{U})$ & - & - & 0.839 & 0.683 & - & - & - & - \\
\hline & SIMLR $(\mathrm{T}+\mathrm{U})$ & - & - & 0.691 & 0.578 & - & - & - & - \\
\hline & BPRMeth-G $(\mathrm{T}+\mathrm{U})$ & - & - & - & - & 0.293 & 0.193 & - & - \\
\hline & Seurat $(\mathrm{T}+\mathrm{S}+\mathrm{U})$ & 0.697 & 0.725 & 0.815 & 0.672 & 0.317 & 0.336 & 0.777 & 0.728 \\
\hline & LIGER $(\mathrm{T}+\mathrm{S}+\mathrm{U})$ & 0.564 & 0.525 & 0.434 & 0.313 & 0.358 & 0.236 & 0.729 & 0.667 \\
\hline & $\mathrm{scACE}(\mathrm{T}+\mathrm{S}+\mathrm{U})$ & 0.859 & 0.855 & 0.490 & 0.371 & 0.042 & 0.016 & 0.496 & 0.479 \\
\hline \multirow{10}{*}{$\begin{array}{c}\text { Source } \\
\text { data }\end{array}$} & couple $\mathrm{CoC}+(\mathrm{T}+\mathrm{S}+\mathrm{U})$ & 0.716 & 0.754 & 0.930 & 0.883 & 0.987 & 0.970 & 0.858 & 0.819 \\
\hline & couple $\mathrm{CoC}(\mathrm{T}+\mathrm{S})$ & 0.666 & 0.731 & 0.930 & 0.883 & 0.985 & 0.965 & 0.858 & 0.819 \\
\hline & $k$-means $(\mathrm{S})$ & 0.682 & 0.729 & 0.268 & 0.190 & 0.972 & 0.939 & - & - \\
\hline & SC3 $(\mathrm{S})$ & 0.464 & 0.562 & 0.953 & 0.908 & 0.987 & 0.968 & - & - \\
\hline & SIMLR (S) & 0.508 & 0.480 & 0.481 & 0.431 & 0.977 & 0.949 & - & - \\
\hline & SHARP $(\mathrm{S})$ & 0.692 & 0.733 & 0.884 & 0.811 & 0.968 & 0.935 & - & - \\
\hline & SAME-clustering (S) & 0.727 & 0.736 & 0.930 & 0.862 & 0.975 & 0.945 & - & - \\
\hline & Seurat $(\mathrm{T}+\mathrm{S}+\mathrm{U})$ & 0.631 & 0.661 & 0.862 & 0.783 & 0.905 & 0.780 & 0.830 & 0.789 \\
\hline & LIGER $(\mathrm{T}+\mathrm{S}+\mathrm{U})$ & 0.889 & 0.859 & -0.029 & 0.027 & 0.842 & 0.748 & 0.800 & 0.731 \\
\hline & $\mathrm{scACE}(\mathrm{T}+\mathrm{S}+\mathrm{U})$ & 0.683 & 0.674 & 0.885 & 0.800 & 0.987 & 0.969 & 0.489 & 0.493 \\
\hline
\end{tabular}

chose the human scRNA-seq data as the source data and chose the mouse scRNA-seq dataset that is sparser as the target data (The proportions of zero entries in the human and mouse scRNA-seq data are $88.90 \%$ and $95.00 \%$, respectively.). The homologs shared by mouse and human are chosen as the linked features, and mouse-specific genes are used as the unlinked features. These data are generated from the drop-seq platform, and their input formats are $\log (\mathrm{UMI}+1)$. We use the cell type annotation (Angelidis et al., 2019) as a benchmark for evaluating the performance of the clustering methods. The optimal number of clusters is $N_{\mathrm{S}}=2$ for the source data, and the values 

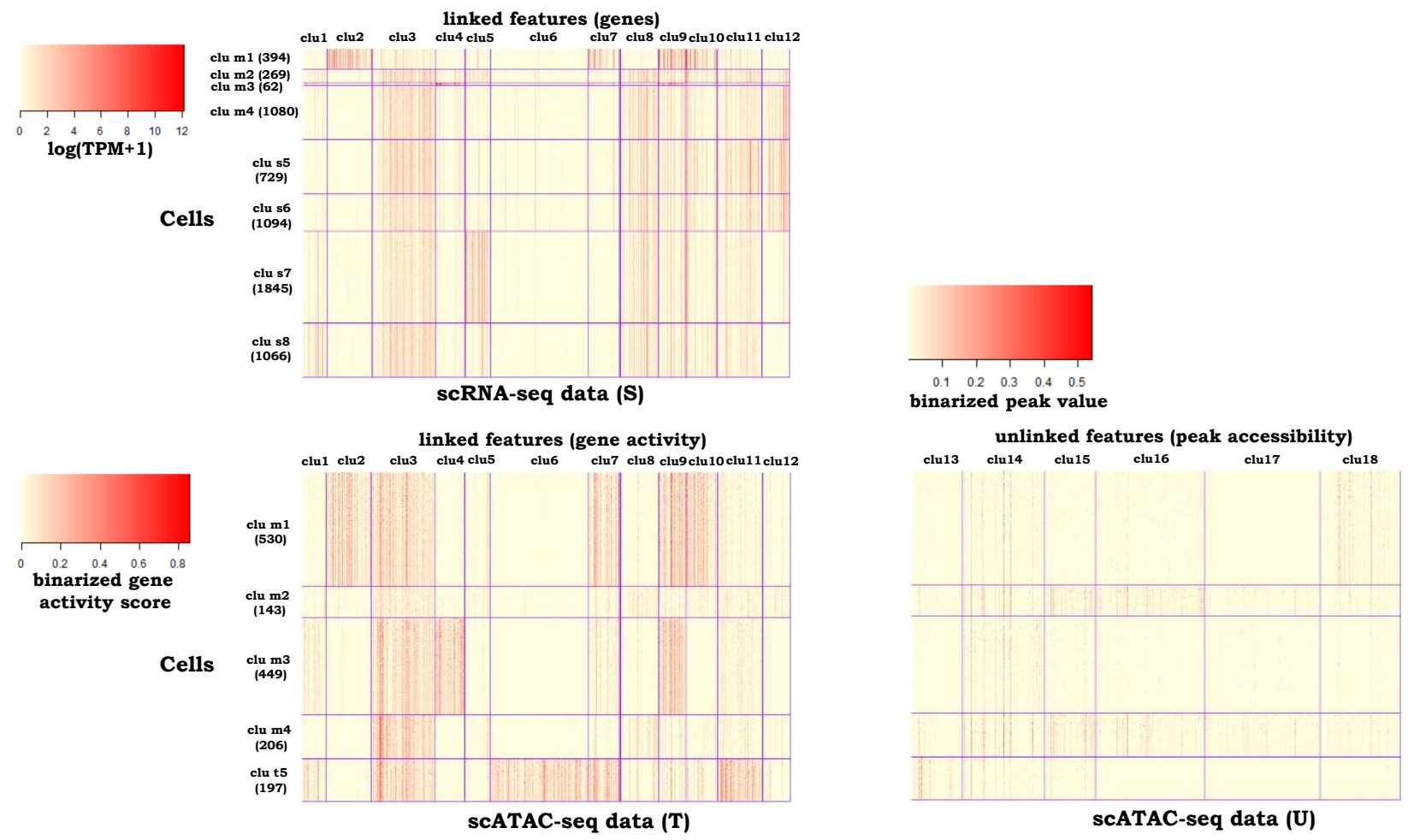

Figure 2: Heatmaps of the clustering results by couple $\mathrm{CoC}+$ for example 1. "clu m" represents the matched cell cluster across the source data and the target data. "clu s" and "clu t" represent the cell clusters that are unique to the source data and the target data, respectively. For better visualization, we randomly averaged every 15 cells within the same cell cluster to generate pseudocells for every heatmap.

of $\mathrm{CH}$ index are close when $N_{\mathrm{T}}=2$ or 3 (Fig.S.7). We chose $N_{\mathrm{T}}=3$, which equals to the true number of cell types. We set the tuning parameters in couple $\mathrm{CoC}+$ as $\lambda=2, \beta=0.04, \gamma=1, K=$ $8, K_{0}=7$ by grid search. We set the number of $N_{\text {sub }}$ as 2 , because the values of the objective function $g\left(N_{\text {sub }}\right)$ for choosing $N_{\text {sub }}$ are smaller when $N_{\text {sub }}=2\left(0.150\right.$ when $N_{\text {sub }}=1$ and 0.077 when $N_{\text {sub }}=2$, respectively).

couple $\mathrm{CoC}+$ performs the best among all the other methods for clustering the target data (Table 1). It improves the performance over CoC by transferring the knowledge from the source data $\mathrm{S}$, and also improves performance over couple $\mathrm{CoC}$ by utilizing the information in the unlinked features. SC3 has the best performance on clustering the source data, and couple $\mathrm{CoC}+$ ranks the second. Compared to couple $\mathrm{CoC}+$, the integrative clustering methods Seurat, LIGER and $s c \mathrm{ACE}$ do not perform well on both source data and target data. Fig. 3 shows the heatmap after clustering by couple $\mathrm{CoC}+$. couple $\mathrm{CoC}+$ clearly clusters similar cells and features. In addition, the patterns of the linked features for the matched clusters tend to be consistent. 


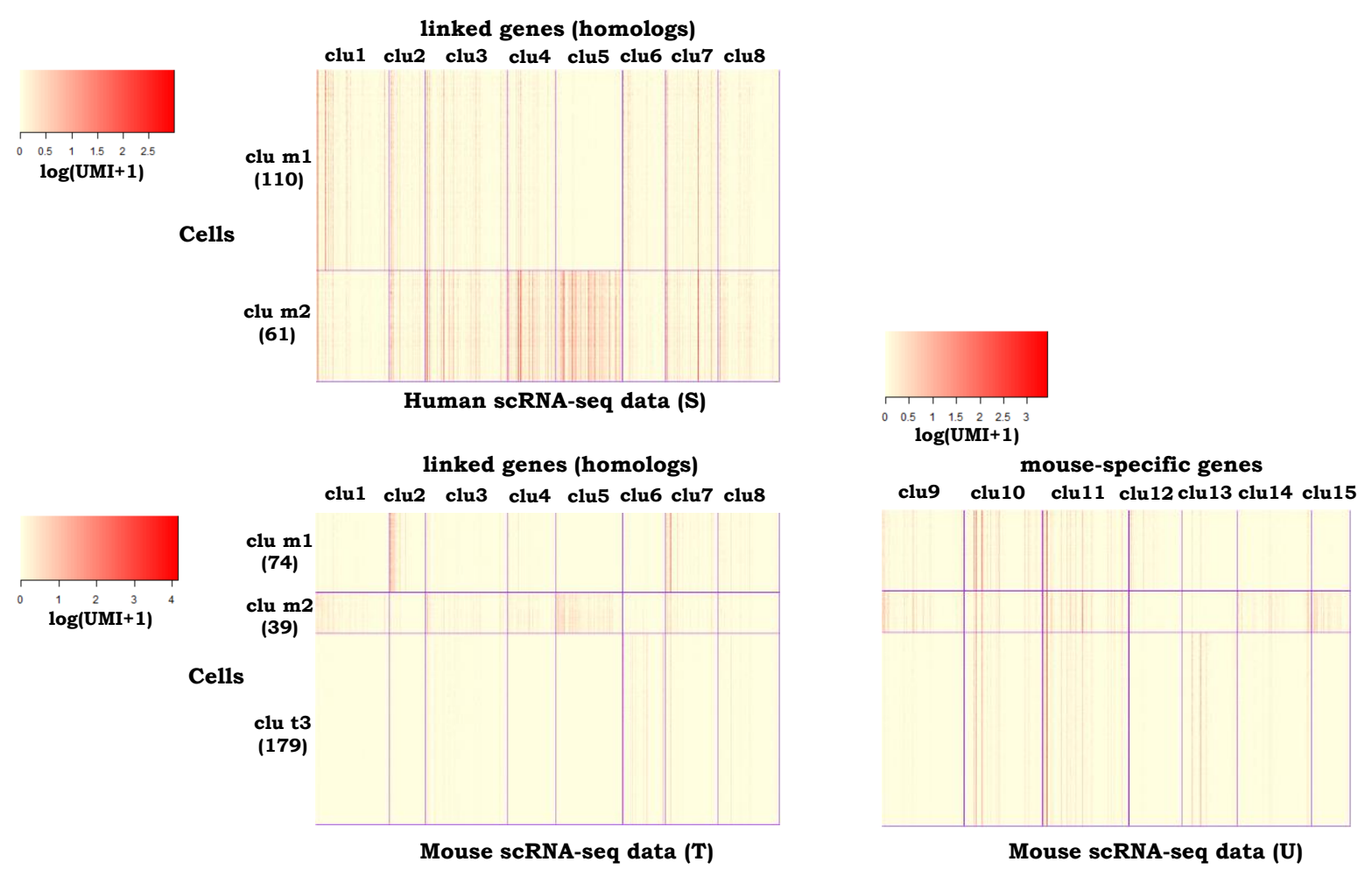

Figure 3: Heatmaps of the clustering results by couple $\mathrm{CoC}+$ for example 2. "clu m" represents the matched cell cluster across the source data and the target data. "clu t" represents the cell cluster that is unique to the target data. For better visualization, we randomly averaged every 15 cells within the same cell cluster to generate pseudocells for every heatmap.

\section{Example 3: integrative clustering for mouse cortex sc-methylation and scRNA-seq data}

In the third example, we evaluated couple $\mathrm{CoC}+$ by jointly clustering sc-methylation data and scRNA-seq data from the mouse cortex (Luo et al., 2017; Tasic et al., 2018). We collected 412 L4 and 690 L2/3 sc-methylation cells, and 1401 L4 and 982 L2/3 IT scRNA-seq cells ("L4" and "L2/3" stand for excitatory neurons in different neocortical layers; IT is the abbreviation of intratelencephalic neuron.). Sc-methylation data tends to be noisier than scRNA-seq data, so we chose sc-methylation as the target data and chose scRNA-seq data as source data. The methylation of gene bodies are the linked features, and the DNA methylation levels at non-CG sites $(\mathrm{mCH}$ levels) for non-overlapping 100kb bins are the unlinked features. We used the provided cell type labels as a benchmark for evaluating the performance of the clustering methods. Fig.S.7 shows that the optimal number of cell clusters are $N_{\mathrm{T}}=2$ and $N_{\mathrm{S}}=2$. We set the tuning parameters in couple $\mathrm{CoC}+$ as $\lambda=0.1, \beta=0.6, \gamma=1, K=5, K_{0}=8$ by grid search. We set the number of matched clusters $N_{\text {sub }}$ as 2 , because the values of the objective function $g\left(N_{\text {sub }}\right)$ for choosing $N_{\text {sub }}$ are smaller when $N_{\text {sub }}=2\left(0.138\right.$ when $N_{\text {sub }}=1$ and 0.061 when $N_{\text {sub }}=2$, respectively).

Table 1 shows that all ten methods have good clustering performance for scRNA-seq data. couple $\mathrm{CoC}+$ performs much better than the other methods for clustering sc-methylation data, 
and it matches well the cell types across the two data types (Table S.1). coupleCoC+ has better clustering performance than $\mathrm{CoC}$, due to the transfer of knowledge from scRNA-seq data to clustering sc-methylation data, and couple $\mathrm{CoC}+$ performs better than couple $\mathrm{CoC}$, because it utilizes the information in the unlinked features while couple CoC does not. The integrative methods Seurat, LIGER and scACE do not perform well on target data. Fig. 4 is the corresponding heatmap after clustering by couple $\mathrm{CoC}+$. For scRNA-seq data, couple CoC + clearly clusters similar cells and features. The signal in sc-methylation data is weaker but we can still see the reverse trend compared with scRNA-seq data: when the gene body methylation level is lower in sc-methylation data, gene expression tends to be higher in scRNA-seq data. The heatmap of the data $U$ further demonstrates the usefulness of including the unlinked features in sc-methylation data, where $\mathrm{mCH}$ levels for non-overlapping 100kb bins better distinguishes the cell types compared with gene body methylation.
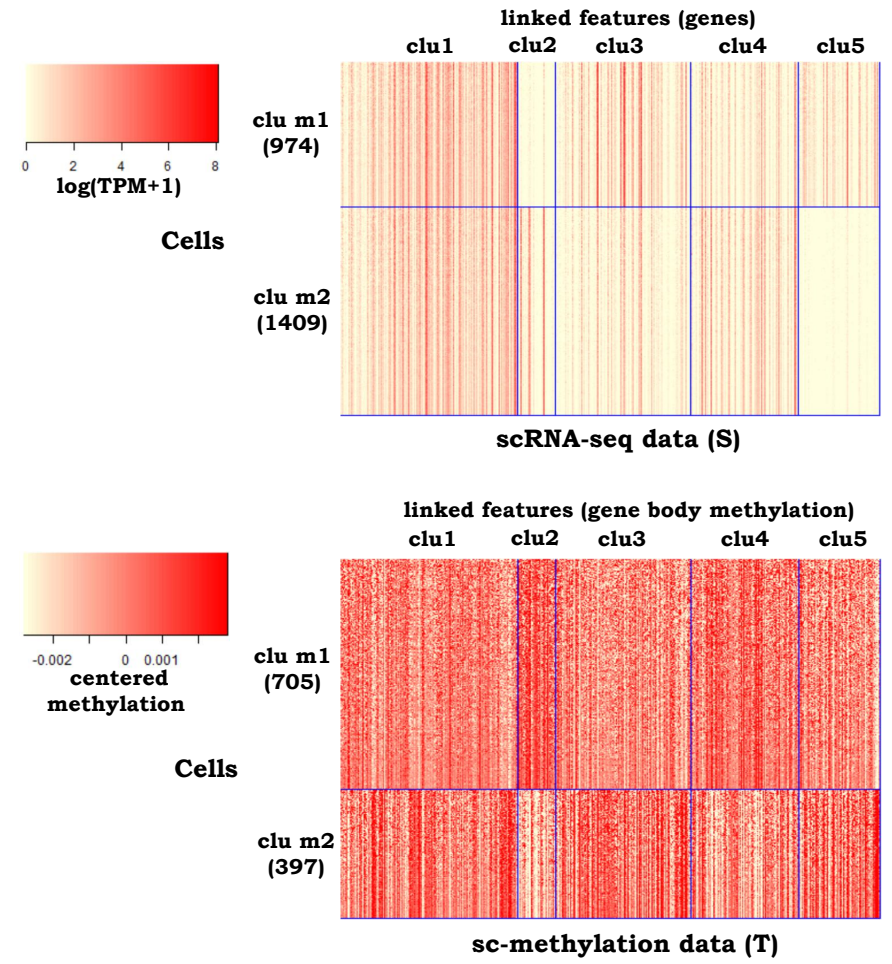
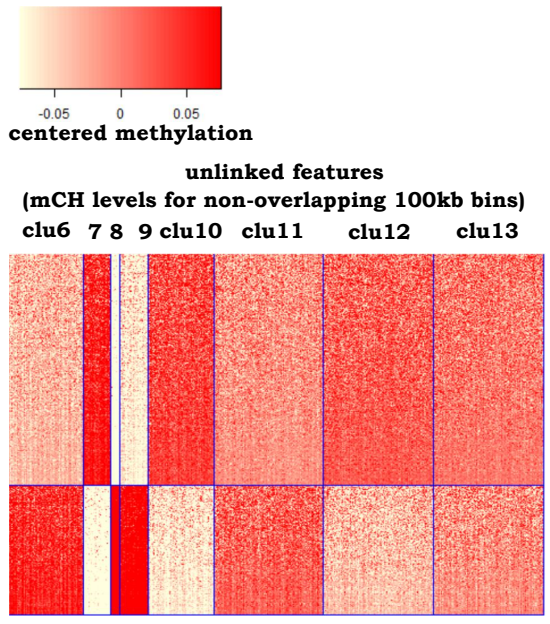

sc-methylation data (U)

Figure 4: Heatmaps of the clustering results by couple $\mathrm{CoC}+$ for example 3. "clu m" represents the matched cell cluster across the source data and the target data. We obtained the centered methylation level by first centering the data matrix by row and then centering the data matrix by column. Grey color in the heatmap of sc-methylation data corresponds to missing data. For better visualization, we randomly averaged every 15 cells within the same cell cluster to generate pseudocells for every heatmap. 


\section{Example 4: integrative clustering for human blood dendritic cells scRNA-seq data from two batches}

In the fourth example, we examined couple $\mathrm{CoC}+$ by integrative clustering of human blood dendritic cell (DC) scRNA-seq data from two batches (Villani et al., 2017). Each batch consists of 96 CD141 DC, 96 CD1C DC, 96 plasmacytoid DC (pDC) and 96 double negative cells. The data were generated from the Smart-Seq2 platform and they were used in a recent benchmark study (Tran et al., 2020). We processed the data similar to Tran et al. (2020), where CD141 DC in batch 1 and CD1C DC in batch 2 were removed. So, both batches share pDC and double negative cells, and each batch has one unshared cell type (CD1C and CD141 respectively) that are biologically similar. We chose batch 1 as the source data and chose batch 2 that is sparser as the target data (The proportions of zero entries in batch 1 and batch 2 are $32.65 \%$ and $42.89 \%$, respectively.). Because all features are shared by source data and target data, and target data have no unlinked features in this example, we set the value of $\beta$ as 0 in objection function (5). We set the number of cell clusters as $N_{\mathrm{T}}=N_{\mathrm{S}}=3$. We set the tuning parameters in coupleCoC + as $\lambda=2, \gamma=1, K=5, K_{0}=5$ by grid search. We set the number of matched clusters $N_{\text {sub }}$ as 2 , because the values of the objective function $g\left(N_{\text {sub }}\right)$ for choosing $N_{\text {sub }}$ are smallest when $N_{\text {sub }}=2$ : $8.66 \times 10^{-4}$ when $N_{\text {sub }}=1,6.37 \times 10^{-4}$ when $N_{\text {sub }}=2$, and $1.08 \times 10^{-3}$ when $N_{\text {sub }}=3$.

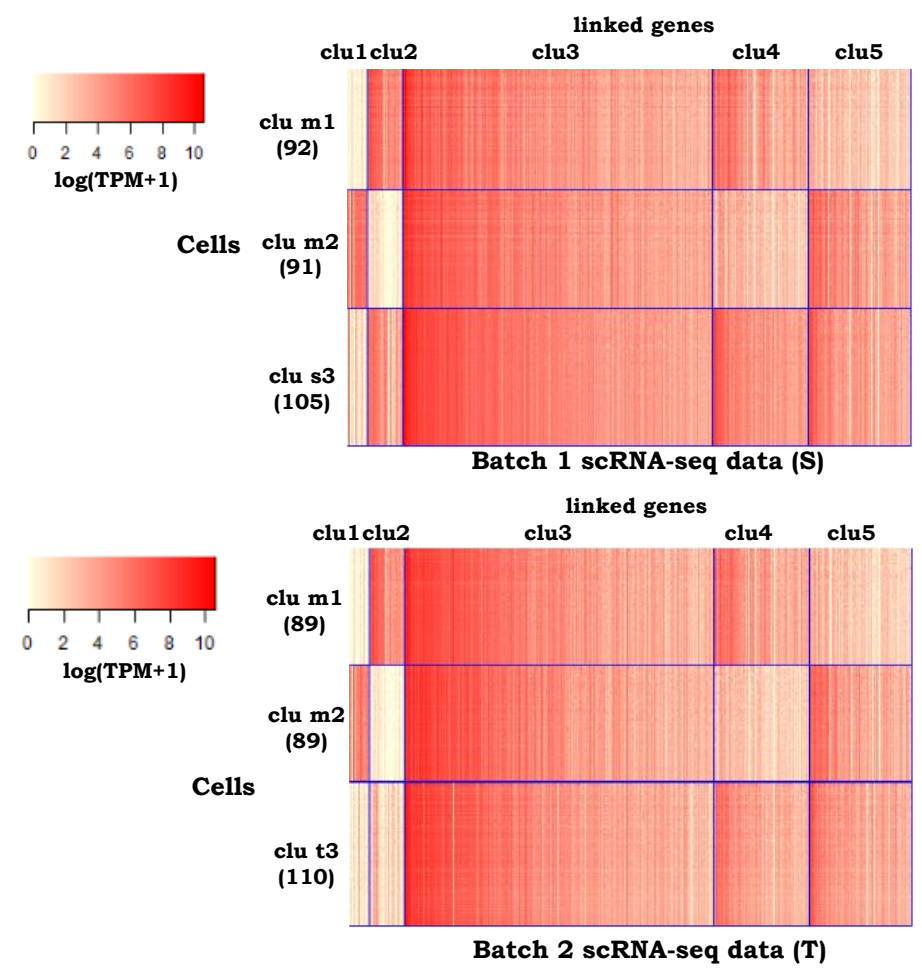

Figure 5: Heatmaps of the clustering results by couple $\mathrm{CoC}+$ for example 4 . "clu m" represents the matched cell cluster across the source data and the target data. "clu s" and "clu t" represent the cell clusters that are unique to the source data and the target data, respectively. For better visualization, we randomly averaged every 15 cells within the same cell cluster to generate pseudocells for every heatmap. 
All five integrative methods, except scACE, have good clustering performance for scRNA-seq data in batch 1 (source data)(Table 1). scACE also fails to cluster scRNA-seq data in batch 2 (target data). Because no unlinked features are included in target data, couple CoC + and couple CoC have similar clustering performance for the data from two batches, and they have competitive performance compared with the other methods (Table 1). Fig. 5 is the corresponding heatmap after clustering by couple $\mathrm{CoC}+$. couple $\mathrm{CoC}+$ clearly clusters similar cells and features, and it accurately found the two matched clusters (the shared pDC and double negative cells) across the two batches (Table S.1.). In addition, the expression patterns for the matched clusters tend to be consistent in the two batches. The cell types unshared by the two batches, including CD1C DC and CD141 DC, are represented by "clu s3" and "clu t3", respectively. They have high similarity with each other, and they are only distinguished by feature cluster "clu2".

\section{Simulation studies}

Lastly, we tested the performance of couple $\mathrm{CoC}+$ through simulation studies. We followed the simulation setup given in Lin et al. (2019) with some modifications specific to our framework. The details for generating data T, S and U are given in Supplementary Text. We set the numbers of cell types in both target data and source data as $N_{\mathrm{T}}=N_{\mathrm{S}}=2$, set the numbers of cells as $n_{\mathrm{T}}=n_{\mathrm{S}}=100$, set the proportion of each cell type as 0.5 and set the number of features as $q=q_{0}=1000$. We varied the differential degree $(w)$ across clusters, the standard deviations $\left(\sigma_{\mathrm{S}}\right.$ and $\sigma_{\mathrm{T}}$, corresponding to source data and target data, respectively) of the generative distribution, and the shift between two means $(d)$ of the generative distributions for the two cell types in data $\mathrm{U}$ (i.e., unlinked features in target data). Larger $w$ leads to better separation of the cell clusters in data $\mathrm{S}$ and data $\mathrm{T}$, larger $\sigma_{\mathrm{S}}$ or $\sigma_{\mathrm{T}}$ leads to higher noise level in data $\mathrm{S}$ or data $\mathrm{T}$, and larger $d$ leads to better separation of the cell clusters in data $\mathrm{U}$. We considered six different simulation settings, varying the parameters $w, \sigma_{\mathrm{S}}, \sigma_{\mathrm{T}}$ and $d$. We compared couple $\mathrm{CoC}+$ with couple $\mathrm{CoC}$ and CoC.

Table 2: The simulation results for clustering the target data in 30 independent runs are summarized. Note that the capital letters in the brackets represent the input data matrices for the corresponding methods: $\mathrm{S}$ represents source data, $\mathrm{T}$ and $\mathrm{U}$ represent the sub-matrices for the linked and unlinked features in target data, respectively. couple $\mathrm{CoC}+$ and couple $\mathrm{CoC}$ utilize both the source data and the target data as input, and they produce clustering results of the cells in source data and target data simultaneously; $\mathrm{CoC}$ is implemented on only target data, and it produces clustering results of the cells in target data. We then summarize the clustering results by calculating ARI and NMI for target data.

\begin{tabular}{|c|c|c|c|c|c|c|c|c|c|c|c|c|}
\hline \multirow{2}{*}{$\begin{array}{c}\text { Clustering } \\
\text { methods }\end{array}$} & \multicolumn{2}{|c|}{$\begin{array}{c}\text { Setting 1 } \\
w=0.67, d=2 \\
\sigma_{\mathrm{S}}=\sigma_{\mathrm{T}}=1.4\end{array}$} & \multicolumn{2}{|c|}{$\begin{array}{c}\text { Setting 2 } \\
w=0.67, d=2 \\
\sigma_{\mathrm{S}}=\sigma_{\mathrm{T}}=1.7\end{array}$} & \multicolumn{2}{|c|}{$\begin{array}{c}\text { Setting 3 } \\
w=0.64, d=2 \\
\sigma_{\mathrm{S}}=\sigma_{\mathrm{T}}=1.4\end{array}$} & \multicolumn{2}{|c|}{$\begin{array}{c}\text { Setting } 4 \\
w=0.64, d=1 \\
\sigma_{\mathrm{S}}=\sigma_{\mathrm{T}}=1.4\end{array}$} & \multicolumn{2}{|c|}{$\begin{array}{c}\text { Setting } 5 \\
w=0.64, d=0 \\
\sigma_{\mathrm{S}}=\sigma_{\mathrm{T}}=1.4\end{array}$} & \multicolumn{2}{|c|}{$\begin{array}{c}\text { Setting } 6 \\
w=0.64, d=2 \\
\sigma_{\mathrm{S}}=2.0, \sigma_{\mathrm{T}}=1.4\end{array}$} \\
\hline & ARI & NMI & ARI & NMI & ARI & NMI & ARI & NMI & ARI & NMI & ARI & NMI \\
\hline couple $\mathrm{CoC}+(\mathrm{S}+\mathrm{T}+\mathrm{U})$ & 0.935 & 0.905 & 0.855 & 0.802 & 0.834 & 0.805 & 0.816 & 0.711 & 0.780 & 0.699 & 0.637 & 0.601 \\
\hline couple $\mathrm{Co}$ & 0.909 & 0.874 & 0.824 & 0.750 & 0.800 & 0.746 & 0.800 & 0.746 & 0.800 & 0.746 & 0.611 & 0.555 \\
\hline $\mathrm{CoC}(\mathrm{T}+\mathrm{U})$ & 0.826 & 0.781 & 0.816 & 0.774 & 0.810 & 0.766 & 0.567 & 0.522 & 0.002 & 0.009 & 0.812 & 0.765 \\
\hline
\end{tabular}

We set the tuning parameters in couple $\mathrm{CoC}+$ as $\lambda=2, \gamma=1, K=3, K_{0}=3$ for all six settings, $\beta=0.1$ for setting 5 , and $\beta=1$ for the remaining settings by grid search. We set 
the number of matched clusters $N_{\text {sub }}$ as 2 . Table 2 presents the simulation results for the target data. In settings 1-3, we fixed $d=2$, and we varied $w, \sigma_{\mathrm{S}}$ and $\sigma_{\mathrm{T}}$. Compared with setting 1 $\left(w=0.67, \sigma_{\mathrm{S}}=\sigma_{\mathrm{T}}=1.4\right)$, setting 2 has higher noise $\left(\sigma_{\mathrm{S}}=\sigma_{\mathrm{T}}=1.7\right)$, and setting 3 has lower differential ability across the cell clusters $(w=0.64)$ in data $\mathrm{T}$ and data $\mathrm{S}$. Compared with setting $3(d=2)$, the unlinked features (data $\mathrm{U})$ in target data have less power in separating the cell types in setting $4(d=1)$. In these four settings, couple $\mathrm{CoC}+$ performs better than couple $\mathrm{CoC}$, because data $\mathrm{U}$ provide information for separating the cell types and couple CoC does not utilize the information in data $\mathrm{U}$. In setting 4 where $d$ is smaller, i.e. data $\mathrm{U}$ have less power in separating the cell types, the margin between couple $\mathrm{CoC}+$ and couple $\mathrm{CoC}$ becomes smaller. Both couple $\mathrm{CoC}+$ and couple $\mathrm{CoC}$ have better clustering performance than $\mathrm{CoC}$, due to the transfer of knowledge from source data to clustering target data. When data U contain no information in separating the cell types (setting $5, d=0$ ), the performance of couple $\mathrm{CoC}+$ is slightly worse than couple CoC. CoC does not work well in setting 5 because it is affected by data $\mathrm{U}$ and it does not transfer knowledge from the source data. When source data $\mathrm{S}$ have higher noise (setting $\left.6, \sigma_{\mathrm{S}}=2.0\right)$, the performance of couple $\mathrm{CoC}+$ and couple $\mathrm{CoC}$ drops and they become inferior to $\mathrm{CoC}$. couple $\mathrm{CoC}+$ is slightly better than couple $\mathrm{CoC}$ in setting 6 , because it incorporates the information in data $\mathrm{U}$.

\section{Convergence and running time}

couple $\mathrm{CoC}+$ is guaranteed to converge as the objective functions in Equations (S.10-S.14) are non-increasing in each iteration. coupleCoC+ tends to converge in 15 iterations (Fig. S.8) for the four real examples. We further summarized the computation time by the methods SC3, SIMLR and couple CoC+ (Table S.4) in each real example. The computation time for clustering source data with $\sim 6.5 \mathrm{~K}$ cells in example 1 are SC3 $=20.52(\mathrm{mins}), \mathrm{SIMLR}=55.50$ (mins). The computation time when we implement couple $\mathrm{CoC}+$ on source data $\mathrm{S}$, target data $\mathrm{T}$ and $\mathrm{U}$ (with a total of $\sim 8.0 \mathrm{~K}$ cells) in example 1 is 28.20 (mins). It shows that couple CoC + has comparable computational speed.

Finally, in order to study the scalability of couple $\mathrm{CoC}+$, we also examined the examples where the number of cells is much larger. We followed the procedures of data generation described in Section: Simulation studies by setting $q=q_{0}=1000$, and generated datasets S, T and U with $n_{\mathrm{S}}+n_{\mathrm{T}}=20 \mathrm{~K}$ and $50 \mathrm{~K}$ cells in total. The computation time by couple CoC + when the total number of cells $n_{\mathrm{S}}+n_{\mathrm{T}}=20 \mathrm{~K}, 50 \mathrm{~K}$ are 163.92 (mins) and 1576.05 (mins), respectively. This demonstrates that couple $\mathrm{CoC}+$ can be implemented on datasets with $20 \mathrm{~K}$ cells, and it can be challenging to implement couple $\mathrm{CoC}+$ on datasets with more than $50 \mathrm{~K}$ cells.

\section{Discussion}

In this research, we demonstrated that couple $\mathrm{CoC}+$, an information-theoretic co-clustering-based unsupervised transfer learning method, is useful in the integrative analysis of single-cell genomic data. First, through clustering and aggregating similar features, couple $\mathrm{CoC}+$ implicitly incorporates dimension reduction of the feature space, which is helpful to reduce the noise in high dimensional single-cell genomic data. We empirically demonstrated that couple $\mathrm{CoC}+$ can alleviate the problems of high dimensionality and sparsity by presenting the clustering results on real single-cell 
genomic datasets. Second, compared with $\mathrm{CoC}$ (Dhillon et al., 2003) and couple CoC (Zeng and Lin, 2020), couple CoC+ yields better clustering results for target data, because it not only transfers knowledge via clustering the features that are linked with the source data but also utilizes information from the unlinked features in target data. Incorporating more information from the target data by including the unlinked features further boosts the clustering performance of the target data. Third, couple $\mathrm{CoC}+$ can automatically find the matched cell subpopulations across source data and target data. Fourth, feature clustering by couple $\mathrm{CoC}+$ is biologically meaningful, where it tends to group genes that are enriched for functional annotation terms closely related to the cell clusters in which they are active. Although our method coupleCoC + has appealing computational speed in clustering the datasets with $\sim 8 \mathrm{~K}$ cells ( $<30$ mins to implement), it is challenging to implement couple $\mathrm{CoC}+$ on very large datasets with more than 50k cells $(>24 \mathrm{hrs}$ to implement). Further improvement in computational speed may be achieved by optimizing the code and developing mini-batch version of the algorithm.

\section{Data and code availability}

All data and source code are publicly available. The processed data (including real data and simulation data) for the input of coupleCoC+ are available at https://github.com/cuhklinlab/ coupleCoC_plus/tree/main/data. The followings are the information for the original unprocessed datasets. The mouse cortex scRNA-seq data in example 1 and example 3 are available at NCBI Gene Expression Omnibus (GEO) under accession GSE115746. The mouse cortex scATACseq data in example 1 were downloaded from https://atlas.gs.washington.edu/mouse-atac/ data/. The mouse and human scRNA-seq data in example 2 are available at https://panglaodb. se/view_data.php?sra=SRA832392\&srs=SRS4237518 and https://panglaodb.se/view_data. php?sra=SRA878024\&srs=SRS4660846, respectively. The mouse cortex sc-methylation data in example 3 are available at GEO under accession GSE97179. The human blood dendritic cells scRNA-seq data in example 4 are available at GEO under accession GSE94820. Source code are available at https://github.com/cuhklinlab/coupleCoC_plus.

\section{Acknowledgements}

We would like to thank Jiaxuan Wangwu for her work on the implement of integrative clustering by LIGER method.

\section{References}

Angelidis, I., Simon, L. M., Fernandez, I. E., Strunz, M., and Mayr, C. H. (2019). An atlas of the aging lung mapped by single cell transcriptomics and deep tissue proteomics. Nat. Commun, 10(963).

Argelaguet, R., Arnol, D., Bredikhin, D., and so on (2020). Mofa+: a statistical framework for comprehensive integration of multi-modal single-cell data. Genome Biol, 21(111). 
Argelaguet, R., Velten, B., Arnol, D., Dietrich, S., Marioni, J. C., and so on (2018). Multi-omics factor analysis-a framework for unsupervised integration of multi-omics data sets. Mol Syst Biol, 14.

Buenrostro, J. D., Wu, B., Litzenburger, U. M., Ruff, D., Gonzales, M. L., Snyder, M. P., and the others (2015). Single-cell chromatin accessibility reveals principles of regulatory variation. Nature, 523:486-90.

Butler, A., Hoffman, P., Smibert, P., Papalexi, E., and Satijia, R. (2018). Integrating singlecell transcriptomic data across different conditions, technologies, and species. Nat Biotechnol, 36(5):411-420.

Calinski, R. B. and Harabasz, J. (1974). A dendrite method for cluster analysis. Communications in Statistics, 3:1-27.

Chen, H., Lareau, C., Andreani, T., and the others (2019). Assessment of computational methods for the analysis of single-cell atac-seq data. Genome biology, 20(1):1-25.

Christopher, D. M., Prabhakar, R., and Hinrich, S. (2008). Introduction to Information Retrieval. Cambridge University Press.

Clark, S. J., Smallwood, S. A., Lee, H. J., Krueger, F., Reik, W., and Kelsey, G. (2017). Genomewide base-resolution mapping of dna methylation in single cells using single-cell bisulfite sequencing (scbs-seq). Nat. Protoc., 12:534-47.

Cover, T. M. and Thomas, J. A. (1991). Elements of information theory. Wiley-Interscience.

Cusanovich, D. A., Daza, R., Adey, A., Pliner, H. A., Christiansen, L., Gunderson, K. L., Steemers, F. J., Trapnell, C., and Shendure, J. (2015). Multiplex single-cell profiling of chromatin accessibility by combinatorial cellular indexing. Science, 348:910-914.

Cusanovich, D. A. and the others (2018). A single-cell atlas of in vivo mammalian chromatin accessibility. Cell, 174:1309-1324.

Dai, W. Y., Yang, Q., Xue, G. R., and Yu, Y. (2008). Self-taught clustering. Proceedings of the 25th international Conference on Machine Learning.

David, L., Johannes, K., Ewa, S., and the others (2020). Eleven grand challenges in single-cell data science. Genome Biol, 21(31).

Dhillon, I. S., Mallela, S., and Modha, D. S. (2003). Information-theoretic co-clustering. Proceedings of the Ninth ACM SIGKDD International Conference on Knowledge Discovery and Data Mining, pages 89-98.

Duren, Z., Chen, X., Zamanighomi, M., Zeng, W., Satpathy, A., Chang, H., Wang, Y., and Wong, W. H. (2018). Integrative analysis of single cell genomics data by coupled non-negative matrix factorizations. Proc. Natl. Acad. Sci., (115):7723-7728. 
Fran, O., Gan, G. M., and Johan, L. M. B. (2019). Panglaodb:a web serer for exploration of mouse and human single-cell rna sequencing data. Database.

Gonzalez-Blas, C. B. et al. (2019). cistopic: cis-regulatory topic modeling on single-cell atac-seq data. Nat. Methods, 16:397-400.

Guo, H., Zhu, P., Wu, X., Li, X., Wen, L., and Tang, F. (2013). Single-cell methylome landscapes of mouse embryonic stem cells and early embryos analyzed using reduced representation bisulfite sequencing. Genome Res., 23:2126-35.

Hicks, S. C., Townes, F. W., Teng, M., and Irizarry, R. A. (2018). Missing data and technical variability in single-cell rna-sequencing experiments. Biostatistics, 19(4):562-578.

Huang, D. W., Sherman, B. T., and Lempicki, R. A. (2009a). Bioinformatics enrichment tools: paths toward the comprehensive functional analysis of large gene lists. Nulceic Acids Res., 37(1):1-13.

Huang, D. W., Sherman, B. T., and Lempicki, R. A. (2009b). Systematic and integrative analysis of large gene lists using david bioinformatics resources. Nature Protoc., 4(1):44-57.

Huh, R., Yang, Y., Jiang, Y., Shen, Y., and Li, Y. (2020). Same-clustering: Single-cell aggregated clustering via mixture model ensemble. Nucleic acids research, 48(1):86-95.

Jaitin, D. A., Kenigsberg, E., Keren-Shaul, H., Elefant, N., Paul, F., Zaretsky, I., Mildner, A., Cohen, N., Jung, S., Tanay, A., et al. (2014). Massively parallel single-cell rna-seq for marker-free decomposition of tissues into cell types. Science, 343:776-779.

Jin, S., Zhang, L., and Nie, Q. (2020). scai: an unsupervised approach for the integrative analysis of parallel single-cell transcriptomic and epigenomic profiles. Genome Biology, 21(25).

Kapourani, C. A. and Sanguinetti, G. (2018). Bprmeth: a flexible bioconductor package for modelling methylation profiles. Bioinformatics, 34:2485-2486.

Kapourani, C. A. and Sanguinetti, G. (2019). Melissa: Bayesian clustering and imputation of single-cell methylomes. Genome Biol, 20(69).

Kharchenko, P. V., Silberstein, L., and Scadden, D. T. (2014). Bayesian approach to single-cell differential expression analysis. Nat. Methods, 11:740-742.

Kiselev, V. Y., Kirschner, K., Schaub, M. T., Andrews, T., Yiu, A., Chandra, T., Natarajan, K. N., Reik, W., Barahona, M., et al. (2017). Sc3: Consensus clustering of single-cell rna-seq data. Nat. Methods, 14(483).

Lafon, S. and Lee, A. B. (2006). Diffusion maps and coarse-graining: a unified framework for dimensionality reduction, graph partitioning, and data set parameterization. IEEE Transactions on Pattern Analysis and Machine Intelligence, 28:1393-1403.

Lin, Z. X., Zamanighomi, M., Daley, T., Ma, S., and Wong, W. H. (2019). Model-based approach to the joint analysis of single-cell data on chromatin accessibility and gene expression. Stat. Sci. 
Lun, A. T. L., Bach, K., and Marioni, J. C. (2016). Pooling across cells to normalize single-cell rna sequencing data with many zero counts. Genome Biol, 15(75).

Luo, C., Keown, C. L., Kurihara, L., Zhou, J., He, Y., Li, J., and the others (2017). Single-cell methylomes identify neuronal subtypes and regulatory elements in mammalian cortex. Science, 357:600-4.

Macaulay, I. C., Ponting, C. P., and Voet, T. (2017). Single-cell multiomics: multiple measurements from single cells. Trends Genet., 33:115-68.

Mezger, A., Klemm, S., Mann, I., Brower, K., Mir, A., Bostick, M., and the others (2018). Highthroughout chromatin accessibility profiling at single-cell resolution. Nat. Commun., 9:34-67.

Rotem, A., Ram, O., Shoresh, N., Sperling, R. A., Goren, A., Weitz, D. A., and Bernstein, B. E. (2015). Single-cell chip-seq reveals cell subpopulations defined by chromatin state. Nat. Biotechnol, 33:1165-1172.

Rozenblatt-Rosen, O., Stubbington, M. J., Regev, A., and Teichmann, S. A. (2017). The human cell atlas: From vision to reality. Nat. News, 550(451).

Sara, B., Itzia, J. F., Agnes, P., and the others (2018). Microglia in neurological diseases: A road map to brain-disease dependent-inflammatory response. Front. Cell. Neurosci.

Schep, N. A., Wu, B., Buenrostro, J. D., and Greenleaf, W. J. (2017). chromvar: inferring transcription-factor-associated accessibility from single-cell epigenomic data. Nat. Methods, 14:975-978.

Smallwood, S. A., Lee, H. J., Angermueller, C., Krueger, F., Saadeh, H., Peat, J., and the others (2014). Single-cell genome-wide bisulfite sequencing for assessing epigenetic heterogeneity. Nat. Methods, 11:817-20.

Stuart, T., Butler, A., Hoffman, P., and the others (2019). Comprehensive integration of single-cell data. Cell, (177):1888-1902.

Sun, Z., Wang, T., Deng, K., Wang, X. F., Lafyatis, R., Ding, Y., Hu, M., and Chen, W. (2017). Dimm-sc: A dirichlet mixture model for clustering droplet-based single cell transcriptomic data. Bioinformatics, (34):139-146.

Tasic, B., Yao, Z., and the others (2018). Shared and distinct transcriptomic cell types across neocortical areas. Nature, 563:72-78.

Tran, H., Ang, K., Chevrier, M., and the others (2020). A benchmark of batch-effect correction methods for single-cell rna sequencing data. Genome Biology, 21(12).

Usoskin, D., Furlan, A., Islam, S., Abdo, H., Lönnerberg, P., Lou, D., Hjerling-Leffler, J., Haeggström, J., Kharchenko, O., Kharchenko, P. V., Linnarsson, S., et al. (2015). Unbiased classification of sensory neuron types by large-scale single-cell rna sequencing. Nat. Neurosci., 18:145-153. 
Vallejos, C. A., Risso, D., Scialdone, A., Dudoit, S., and Marioni, J. C. (2017). Normalizing single-cell rna sequencing data: challenges and opportunities. Nat. Methods, 14:565-571.

Vandermaaten, L. (2008). Visualizing data using t-sne. J. Mach. Learn. Res., 9:2579-2605.

Villani, A.-C., Satija, R., Reynolds, G., and the others (2017). Single-cell rna-seq reveals new types of human blood dendritic cells, monocytes, and progenitors. Science, 356(6335).

Wan, S., Kim, J., and Won, K. J. (2020). Sharp: hyperfast and accurate processing of single-cell rna-seq data via ensemble random projection. Genome Research, 30(2):205-213.

Wang, B., Zhu, J., Pierson, E., Ramazzotti, D., and Batzoglou, S. (2017). Visualization and analysis of single-cell rna-seq data by kernel-based similarity learning. Nat. Methods, 14:414416.

Welch, J. D., Kozareva, V., Ferreira, A., Vanderburg, C., and the others (2019). Single-cell multiomic integration compares and contrasts features of brain cell identity. Cell, 177(7):1873-1887.

Xiong, L., Xu, K., Tian, K., Shao, Y., Tang, L., Gao, G., Zhang, M., Jiang, T., and Zhang, Q. C. (2019). Scale method for single-cell atac-seq analysis via latent feature extraction. Nat. Commun, 10(4576).

Yang, Y., Huh, R., Culpepper, H. W., Lin, Y., Love, M. I., and Li, Y. (2018). Safe-clustering: Single-cell aggregated(from ensemble)clustering for single-cell rna-seq data. Bioinformatics.

Zamanighomi, M., Lin, Z., Daley, T., Chen, X., Duren, Z., Schep, A., Greenleaf, W. J., and Wong, W. H. (2018). Unsupervised clustering and epigenetic classification of single cells. Nat. Commun, $9(2410)$.

Zeng, P. and Lin, Z. (2020). Coupled co-clustering-based unsupervised transfer learning for the ingetrative analysis of single-cell genomics data. Briefings in bioinformatics.

Zhang, H., Lee, C. A. A., Li, Z., and the others (2018). A multitask clustering approach for single-cell rna-seq analysis in recessive dystrophic epidermolysis bullosa. PLoS Comput Biol, $14(4)$.

Zhang, L. and Nie, Q. (2021). scmc learns biological variation through the alignment of multiple single-cell genomics datasets. Genome Biology, 22(10).

Zhu, L., Lei, J., Klei, L., Devlin, B., and Roeder, K. (2019). Semisoft clustering of single-cell data. Proc. Natl. Acad. Sci. USA, 116:466-471. 